Research Article

\title{
A note on Chebyshev inequality via $k$-generalized fractional integrals
}

\author{
Juan E. Nápoles Valdés ${ }^{1,2, *, \dagger}$, Florencia Rabossi ${ }^{1}$ \\ ${ }^{1}$ Facultad de Ciencias Exactas y Naturales y Agrimensura, Universidad Nacional del Nordeste, Av. Libertad 5450, Corrientes, Argentina \\ ${ }^{2}$ Facultad Regional Resistencia, Universidad Tecnologica Nacional, French 414, Resistencia, Chaco, Argentina
}

(Received: 1 May 2021. Received in revised form: 27 July 2021. Accepted: 29 July 2021. Published online: 3 August 2021.)

(c) 2021 the authors. This is an open access article under the CC BY (International 4.0) license (www.creativecommons.org/licenses/by/4.0/).

\begin{abstract}
In this paper, by using the $k$-generalized fractional integrals, we establish certain integral inequalities for the Chebyshev functional in case of synchronous function. The obtained inequalities generalize several known integral inequalities.
\end{abstract}

Keywords: generalized $k$-proportional fractional integral operators with general kernel; Chebyshev inequality; synchronous functions.

2020 Mathematics Subject Classification: 26A33, 26D10, 47A63.

\section{Introduction}

One of the most developed mathematical areas in recent years is that of integral inequalities, particularly involving various fractional and generalized integral operators; for example, see [1, 5-8, 10,15, 17, 18, 21, 25, 31, 43, 44]. Recently, the generalized $k$-proportional fractional integral operators with general kernel were defined, which contain many of the known fractional operators. In order to give detail of this work, we need to first present some preliminary results.

Throughout this paper, we use the functions $\Gamma$ (see $[33,36,46,47])$ and $\Gamma_{k}$ (see [13]) defined as follows:

$$
\Gamma(z)=\int_{0}^{\infty} \tau^{z-1} e^{-\tau} \mathrm{d} \tau, \quad \Re(z)>0
$$

and

$$
\Gamma_{k}(z)=\int_{0}^{\infty} \tau^{z-1} e^{-\tau^{k} / k} \mathrm{~d} \tau, k>0 .
$$

It is clear that if $k \rightarrow 1$ we have $\Gamma_{k}(z) \rightarrow \Gamma(z), \Gamma_{k}(z)=(k)^{\frac{z}{k}-1} \Gamma\left(\frac{z}{k}\right)$ and $\Gamma_{k}(z+k)=z \Gamma_{k}(z)$. Also, we define the $k$-beta function as follows

Notice that

$$
B_{k}(u, v)=\frac{1}{k} \int_{0}^{1} \tau^{\frac{u}{k}-1}(1-\tau)^{\frac{v}{k}-1} d \tau
$$

and

$$
B_{k}(u, v)=\frac{1}{k} B\left(\frac{u}{k}, \frac{v}{k}\right)
$$

$$
B_{k}(u, v)=\frac{\Gamma_{k}(u) \Gamma_{k}(v)}{\Gamma_{k}(u+v)} .
$$

The fractional integral operator of Riemann-Liouville are being extended and generalized in various ways. One of such ways are presented in this paper. From the point of view of differential operators and by manipulating simple algebraic identities, one can follow the idea of fractional differential operators of the Riemann-Liouville or Caputo type. From the simple facts $\alpha=1+\alpha-1$ and $\alpha=\alpha-1+1$ we have

$$
{ }^{R L} D^{\alpha} f(t)=\frac{d}{d t}\left\{J_{a_{1}}^{1-\alpha}(f)(t)\right\},
$$

and

$$
{ }^{C} D^{\alpha} f(t)=J_{a_{1}}^{1-\alpha}\left(\frac{d f}{d t}\right)(t),
$$

respectively.

Next, we present several definitions of fractional integrals, some very recent (with $0 \leq a_{1}<\tau<a_{2} \leq \infty$ ). One of the first operators that can be called fractional is the Riemann-Liouville fractional derivative of order $\alpha \in \mathbb{C}$, with $\operatorname{Re}(\alpha)>0$, defined as follows (see [16]). 
Definition 1.1. Let $a_{1}<a_{2}$ and $f \in L^{1}\left(\left(a_{1}, a_{2}\right) ; \mathbb{R}\right)$. The right and left side Riemann-Liouville fractional integrals of order $\alpha$, with $\operatorname{Re}(\alpha)>0$, are defined, respectively, by

$$
R L J_{a_{1}+}^{\alpha} f(t)=\frac{1}{\Gamma(\alpha)} \int_{a_{1}}^{t}(t-s)^{\alpha-1} f(s) d s,
$$

and

$$
{ }^{R L} J_{a_{2}-}^{\alpha} f(t)=\frac{1}{\Gamma(\alpha)} \int_{t}^{a_{2}}(s-t)^{\alpha-1} f(s) d s,
$$

with $t \in\left(a_{1}, a_{2}\right)$.

Other definitions of fractional integral operators are the following ones.

Definition 1.2. [19] Let $a_{1}<a_{2}$ and $f \in L^{1}\left(\left(a_{1}, a_{2}\right) ; \mathbb{R}\right)$. The right and left side Hadamard fractional integrals of order $\alpha$, with $\operatorname{Re}(\alpha)>0$, are defined, respectively, by

$$
H_{a_{1}+}^{\alpha} f(t)=\frac{1}{\Gamma(\alpha)} \int_{a_{1}}^{t}\left(\log \frac{t}{s}\right)^{\alpha-1} \frac{f(s)}{s} d s,
$$

and

$$
H_{a_{2}-}^{\alpha} f(t)=\frac{1}{\Gamma(\alpha)} \int_{t}^{a_{2}}\left(\log \frac{s}{t}\right)^{\alpha-1} \frac{f(s)}{s} d s,
$$

with $t \in\left(a_{1}, a_{2}\right)$.

In [22], the author introduced new fractional integral operators, called the Katugampola fractional integrals, in the following way.

Definition 1.3. Let $0<a_{1}<a_{2}, f:\left[a_{1}, a_{2}\right] \rightarrow \mathbb{R}$ be an integrable function, and $\alpha \in(0,1), \rho>0$ be two fixed real numbers. The right and left side Katugampola fractional integrals of order $\alpha$ are defined, respectively, by

$$
K_{a_{1}+}^{\alpha, \rho} f(t)=\frac{\rho^{1-\alpha}}{\Gamma(\alpha)} \int_{a_{1}}^{t} \frac{s^{\rho-1}}{\left(t^{\rho}-s^{\rho}\right)^{1-\alpha}} f(s) d s,
$$

and

$$
K_{a_{2}-}^{\alpha, \rho} f(t)=\frac{\rho^{1-\alpha}}{\Gamma(\alpha)} \int_{t}^{a_{2}} \frac{t^{\rho-1}}{\left(s^{\rho}-t^{\rho}\right)^{1-\alpha}} f(s) d s,
$$

with $t \in\left(a_{1}, a_{2}\right)$.

The left-sided and right-sided Riemann-Liouville $k$-fractional integrals are given in [28].

Definition 1.4. Let $f \in L_{1}\left[a_{1}, a_{2}\right]$. Then the Riemann-Liouville $k$-fractional integrals of order $\alpha \in \mathbb{C}, \Re(\alpha)>0$ and $k>0$ are given by the expressions:

$$
\begin{array}{ll}
{ }^{\alpha} I_{a_{1}+}^{k} f(u)=\frac{1}{k \Gamma_{k}(\alpha)} \int_{a_{1}}^{u}(u-\tau)^{\frac{\alpha}{k}-1} f(\tau) d \tau, & u>a_{1}, \\
{ }^{\alpha} I_{a_{2}-}^{k} f(u)=\frac{1}{k \Gamma_{k}(\alpha)} \int_{u}^{a_{2}}(\tau-u)^{\frac{\alpha}{k}-1} f(\tau) d \tau, \quad u<a_{2} .
\end{array}
$$

A more general definition of the Riemann-Liouville fractional integrals is given in [24].

Definition 1.5. Let $f:\left[a_{1}, a_{2}\right] \rightarrow \mathbb{R}$ be an integrable function. Also, let $g$ be an increasing and positive function on $\left(a_{1}, a_{2}\right]$ with a continuous derivative $g^{\prime}$ on $\left(a_{1}, a_{2}\right)$. The left and right sided fractional integrals of a function $f$ with respect to another function $g$ on $\left[a_{1}, a_{2}\right]$ of order $\alpha \in \mathbb{C}, \Re(\alpha)>0$, are expressed by:

$$
\begin{aligned}
& { }_{g}^{\alpha} I_{a_{1}^{+}} f(u)=\frac{1}{\Gamma(\alpha)} \int_{a_{1}}^{u}(g(u)-g(\tau))^{\alpha-1} g^{\prime}(\tau) f(\tau) d \tau, \quad u>a_{1}, \\
& { }_{g}^{\alpha} I_{a_{2}^{-}} f(u)=\frac{1}{\Gamma(\alpha)} \int_{u}^{a_{2}}(g(\tau)-g(u))^{\alpha-1} g^{\prime}(\tau) f(\tau) d \tau, \quad u<a_{2} .
\end{aligned}
$$

A $k$-fractional analogue of Definition 1.5 is given in the following (see [4,26,37]). 
Definition 1.6. Let $f:\left[a_{1}, a_{2}\right] \rightarrow \mathbb{R}$ be an integrable function. Also, let $g$ be an increasing and positive function on $\left(a_{1}, a_{2}\right]$ with a continuous derivative $g^{\prime}$ on $\left(a_{1}, a_{2}\right)$. The left and right sided $k$-fractional integrals of a function $f$ with respect to another function $g$ on $\left[a_{1}, a_{2}\right]$ of order $\alpha \in \mathbb{C}, \Re(\alpha)>0$ and $k>0$ are expressed by:

$$
\begin{aligned}
& { }^{\alpha} I_{a_{1}^{+}}^{k} f(u)=\frac{1}{k \Gamma_{k}(\alpha)} \int_{a_{1}}^{u}(g(u)-g(\tau))^{\frac{\alpha}{k}-1} g^{\prime}(\tau) f(\tau) d \tau, \quad u>a_{1}, \\
& { }^{\alpha} I_{a_{2}^{-}}^{k} f(u)=\frac{1}{k \Gamma_{k}(\alpha)} \int_{u}^{a_{2}}(g(\tau)-g(u))^{\frac{\alpha}{k}-1} g^{\prime}(\tau) f(\tau) d \tau, \quad u<a_{2} .
\end{aligned}
$$

Next, we have the definition of the generalized proportional fractional (GPF) integral operator (see [38]).

Definition 1.7. Let $U \in X_{\Psi}^{q}(0,+\infty), 0<a_{1}<a_{2}$, and there be an increasing and positive monotone function $\Psi$ defined on $[0,+\infty)$ having continuous derivative $\Psi^{\prime}$ on $[0,+\infty)$ with $\Psi(0)=0$. Then the left-sided and right-sided GPF-integral operator of a function $U$ in the sense of another function $\Psi$ of order $\eta$ are stated as:

$$
{ }^{\Psi} T_{v_{1}+}^{\eta, \varsigma} U(\varsigma)=\frac{1}{\varsigma^{\eta} \Gamma(\eta)} \int_{v_{1}}^{\varsigma} \frac{\exp \left[\frac{\varsigma-1}{\varsigma}(\Psi(\varsigma)-\Psi(\xi))\right]}{(\Psi(\varsigma)-\Psi(\xi))^{1-\eta}} U(\xi) \Psi^{\prime}(\xi) d \xi, \quad v_{1}<\varsigma,
$$

and

$$
{ }^{\Psi} T_{v_{2}-}^{\eta, \varsigma} U(\varsigma)=\frac{1}{\varsigma^{\eta} \Gamma(\eta)} \int_{v_{1}}^{\varsigma} \frac{\exp \left[\frac{\varsigma-1}{\varsigma}(\Psi(\xi)-\Psi(\varsigma))\right]}{(\Psi(\xi)-\Psi(\varsigma))^{1-\eta}} U(\xi) \Psi^{\prime}(\xi) d \xi, \quad \varsigma<v_{2},
$$

where the proportionality index $\varsigma \in(0,1], \eta \in \mathbb{C}, \operatorname{Re}(\eta)>0$, and $\Gamma$ is the gamma function.

The functional space on which we develop our work is the following.

Definition 1.8. Let $h \in L_{1}[0,+\infty)$ and $F$ be a continuous and positive function on $[0,+\infty)$ with $F(0)=0$. The space $X_{F}^{q}(0,+\infty)(1 \leq q<+\infty)$ consists of those real-valued Lebesgue measurable functions $h$ on $[0,+\infty)$ for which

$$
\|h\|_{X_{F}^{q}}=\left(\int_{a_{1}}^{a_{2}}|h(s)|^{q} F(s) d s\right)^{\frac{1}{q}}<+\infty, \quad 1 \leq q<+\infty,
$$

and for the case $q=+\infty$

$$
\|h\|_{X_{F}^{\infty}}=e s s \sup _{0 \leq s<\infty}[F(s) h(s)] .
$$

We are now in a position to define the generalized integral operators that we use in our work (see [30]).

Definition 1.9. Let $h \in X_{F}^{q}(0,+\infty)$ and $F$ be a continuous and positive function on $[0,+\infty)$ with $F(0)=0$. The right and left side generalized $k$-proportional fractional integral operators with general kernel of order $\gamma$ of $h$ are defined, respectively, by

$$
J_{F, a_{1}+}^{\frac{\gamma}{k}, \lambda} h(\chi)=\frac{1}{\lambda^{\gamma} k \Gamma_{k}(\gamma)} \int_{a_{1}}^{\chi} \frac{G\left(\mathbb{F}_{+}(\chi, s), \lambda\right) F(s) h(s)}{\left(\mathbb{F}_{+}(\chi, s)\right)^{1-\frac{\gamma}{k}}} d s,
$$

and

$$
J_{F, a_{2}-}^{\frac{\gamma}{k}, \lambda} h(\chi)=\frac{1}{\lambda^{\gamma} k \Gamma_{k}(\gamma)} \int_{\chi}^{a_{2}} \frac{G\left(\mathbb{F}_{-}(s, \chi), \lambda\right) F(s) h(s)}{\left(\mathbb{F}_{-}(s, \chi)\right)^{1-\frac{\gamma}{k}}} d s,
$$

where the proportionality index $\lambda \in(0,1), \gamma \in \mathbb{C}, \operatorname{Re}(\gamma)>0, \chi \in\left(a_{1}, a_{2}\right)$,

$$
\begin{aligned}
& \mathbb{F}_{+}(\chi, s)=\int_{s}^{\chi} F(r) d r, \\
& \mathbb{F}_{-}(s, \chi)=\int_{\chi}^{s} F(r) d r,
\end{aligned}
$$

and

$$
G\left(\mathbb{F}_{+}(\chi, s), 1\right)=G\left(\mathbb{F}_{-}(\chi, s), 1\right)=1 .
$$

Of course there are other integral fractional operators and their variations can be considered, however we do not discuss them here.

Remark 1.1. Next, we will show how many integral operators are particular cases of (1) and (2).

1. If in Definition 1.9 we take $k=1, F=1$, and $\lambda=1$, we obtain the Riemann-Liouville operators given in Definition 1.1.

2. Under the above conditions, if $k \neq 1$ then, from Definition 1.9, the $k$-fractional operators defined in [28] are obtained. 
3. If $F(s)=\frac{1}{s}, \lambda=1$ and $k=1$, then the Hadamard fractional operator is reproduced, see Definition 1.2, and [19,23,39].

4. If $F(s)=\frac{1}{s^{\rho}}, \lambda=1$, and $k=1$, then we obtain the Katugampola's fractional operator given in Definition 1.3, see [22].

5. The Choices $\lambda=1, F(s)=g^{\prime}(s)$, and $k=1$, lead us to the integral operator defined in [24].

6. If we take $F(s)=\frac{1}{s}, k \neq 1$ and

$$
G\left(\mathbb{F}_{+}(\chi, s), \lambda\right)=\exp \left[\frac{\lambda-1}{\lambda}\left(\ln \frac{\chi}{s}\right)\right]
$$

then we obtain the integral operator defined in [35].

7. We can obtain an integral operator with a non-singular nucleus, of the Riemann-Liouville type, by putting $\gamma=k=1$, $F(t)=1$, and

$$
G\left(\mathbb{F}_{+}(x, s), \alpha\right)=\exp \left[-\frac{1-\alpha}{\alpha}(x-s)\right],
$$

that is a slight modification of the operator defined in [3].

8. If we Choose $\lambda \neq 1, F(s)=g^{\prime}(s), k=1$ and

$$
G\left(\mathbb{F}_{+}(\chi, s), \lambda\right)=\exp \left[\frac{\lambda-1}{\lambda}(g(\chi)-g(s))\right],
$$

then we obtain the integral operator defined in [38], known as GFP and is given in Definition 1.7.

\section{Results}

One of the best known integral inequalities is Chebyshev's inequality (see [11]), which establishes relationships between the integral of the product of two functions and the product of their integrals. This inequality is stated in the framework of the classical Riemann integral:

$$
\frac{1}{b-a} \int_{a}^{b} f(x) g(x) d x \geq\left(\frac{1}{b-a} \int_{a}^{b} f(x) d x\right)\left(\frac{1}{b-a} \int_{a}^{b} g(x) d x\right),
$$

where $f$ and $g$ are two integrable and synchronous functions on $[a, b], a<b, a, b \in \mathbb{R}$. Inequality (3) has many applications in diverse research subjects such as numerical quadrature, transform theory, probability, existence of solutions of differential equations and statistical problems. Many authors have investigated generalizations of the Chebyshev's inequality (3), which are called Chebyshev type inequalities (for example, see [2,9,12,14,20,27, 29, 32,34,41-43, 45]). This inequality is generalized also in the present paper by using the generalized operator of Definition 1.9, which contain many inequalities reported in the literature as particular cases.

Definition 2.1. Two functions $f$ and $g$ are said to synchronous (asynchronous) on $[0, \infty)$, if

$$
((f(u)-f(v))(g(u)-g(v))) \geq(\leq) 0,
$$

for all $u, v \in[0,+\infty)$.

In Chebyshev's work cited above, the following functional is presented, which has been the subject of researches:

$$
T(f, g)=\frac{1}{b-a} \int_{a}^{b} f(x) g(x) d x-\left(\frac{1}{b-a} \int_{a}^{b} f(x) d x\right)\left(\frac{1}{b-a} \int_{a}^{b} g(x) d x\right),
$$

where $f$ and $g$ are two integrable functions which are synchronous on $[a, b]$.

In our work, we consider the following generalization of the functional $T(f, g)$ (see (4)):

$$
C_{\lambda}^{\frac{\gamma}{k}}(f, g)=J_{F, a_{1}+}^{\frac{\gamma}{k}, \lambda}(f g)(\chi)-\left(\frac{J_{F, a_{1}+}^{\frac{\gamma}{k}, \lambda} f(\chi) J_{F, a_{1}+}^{\frac{\gamma}{k}, \lambda} g(\chi)}{J_{F, a_{1}+}^{\frac{\gamma}{k}, \lambda}(1)(\chi)}\right)
$$

with

$$
J_{F, a_{1}+}^{\frac{\gamma}{k}, \lambda}(1)(\chi)=\frac{1}{\lambda^{\gamma} k \Gamma_{k}(\gamma)} \int_{a_{1}}^{\chi} \frac{G\left(\mathbb{F}_{+}(\chi, u), \lambda\right) F(u)(1) d u}{\left(\mathbb{F}_{+}(\chi, u)\right)^{1-\frac{\gamma}{k}}} .
$$

Our first result is given in the form of following theorem. 
Theorem 2.1. Let $f$ and $g$ be two synchronous on $[0, \infty)$. Then for all $\chi>a_{1} \geq 0, \alpha>0, \lambda \in(0,1)$, and $\gamma \in \mathbb{C}$ with $\operatorname{Re}(\gamma)>0$, it holds that

$$
C_{\lambda}^{\frac{\gamma}{k}}(f, g) \geq 0
$$

Proof. As $f$ and $g$ are synchronous functions on $[0, \infty)$, it follows that

$$
(f(u)-f(v))(g(u)-g(v)) \geq 0,
$$

for all $u, v \geq 0$. Therefore,

$$
f(u) g(u)+f(v) g(v) \geq f(u) g(v)+f(v) g(u) .
$$

Multiplying both sides of (5) by

$$
\frac{1}{\lambda^{\gamma} k \Gamma_{k}(\gamma)} \frac{G\left(\mathbb{F}_{+}(\chi, u), \lambda\right) F(u)}{\left(\mathbb{F}_{+}(\chi, u)\right)^{1-\frac{\gamma}{k}}}
$$

and integrating the result with respect to $u$ over $\left(a_{1}, \chi\right)$, we get

$$
\begin{aligned}
& \frac{1}{\lambda^{\gamma} k \Gamma_{k}(\gamma)} \int_{a_{1}}^{\chi} \frac{G\left(\mathbb{F}_{+}(\chi, u), \lambda\right) F(u) f(u) g(u) d u}{\left(\mathbb{F}_{+}(\chi, u)\right)^{1-\frac{\gamma}{k}}}+\frac{f(v) g(v)}{\lambda^{\gamma} k \Gamma_{k}(\gamma)} \int_{a_{1}}^{\chi} \frac{G\left(\mathbb{F}_{+}(\chi, u), \lambda\right) F(u) d u}{\left(\mathbb{F}_{+}(\chi, u)\right)^{1-\frac{\gamma}{k}}} \\
\geq & \frac{g(v)}{\lambda^{\gamma} k \Gamma_{k}(\gamma)} \int_{a_{1}}^{\chi} \frac{G\left(\mathbb{F}_{+}(\chi, u), \lambda\right) f(u) F(u) d u}{\left(\mathbb{F}_{+}(\chi, u)\right)^{1-\frac{\gamma}{k}}}+\frac{f(v)}{\lambda^{\gamma} k \Gamma_{k}(\gamma)} \int_{a_{1}}^{\chi} \frac{G\left(\mathbb{F}_{+}(\chi, u), \lambda\right) g(u) F(u) d u}{\left(\mathbb{F}_{+}(\chi, u)\right)^{1-\frac{\gamma}{k}}} .
\end{aligned}
$$

We can write

$$
J_{F, a_{1}+}^{\frac{\gamma}{k}, \lambda}(f g)(\chi)+f(v) g(v) J_{F, a_{1}+}^{\frac{\gamma}{k}, \lambda}(1)(\chi) \geq g(v) J_{F, a_{1}+}^{\frac{\gamma}{k}, \lambda}(f)(\chi)+f(v) J_{F, a_{1}+}^{\frac{\gamma}{k}, \lambda}(g)(\chi) .
$$

Multiplying both sides of (6) by

$$
\frac{1}{\lambda^{\gamma} k \Gamma_{k}(\gamma)} \frac{G\left(\mathbb{F}_{+}(\chi, v), \lambda\right) F(v)}{\left(\mathbb{F}_{+}(\chi, v)\right)^{1-\frac{\gamma}{k}}}
$$

then integrating the resulting inequality with respect to $v$ over $\left(a_{1}, \chi\right)$, we obtain

$$
\begin{aligned}
& J_{F, a_{1}+}^{\frac{\gamma}{k}, \lambda}(1)(\chi) J_{F, a_{1}+}^{\frac{\gamma}{k}, \lambda}(f g)(\chi)+J_{F, a_{1}+}^{\frac{\gamma}{k}, \lambda}(f g)(\chi) J_{F, a_{1}+}^{\frac{\gamma}{k}, \lambda}(1)(\chi) \\
\geq & J_{F, a_{1}+}^{\frac{\gamma}{k}, \lambda}(g)(\chi) J_{F, a_{1}+}^{\frac{\gamma}{k}, \lambda}(f)(\chi)+J_{F, a_{1}+}^{\frac{\gamma}{k}, \lambda}(f)(\chi) J_{F, a_{1}+}^{\frac{\gamma}{k}, \lambda}(g)(\chi)
\end{aligned}
$$

that is,

$$
J_{F, a_{1}+}^{\frac{\gamma}{k}, \lambda}(1)(\chi) J_{F, a_{1}+}^{\frac{\gamma}{k}, \lambda}(f g)(\chi) \geq J_{F, a_{1}+}^{\frac{\gamma}{k}, \lambda}(g)(\chi) J_{F, a_{1}+}^{\frac{\gamma}{k}, \lambda}(f)(\chi) .
$$

Reordering it and taking into account the definition of $C_{\lambda}^{\frac{\gamma}{k}}(f, g)$, the desired inequality is obtained. This completes the proof.

Remark 2.1. If we take $k=1, a_{1}=1$ and $F(s)=\frac{1}{s}$ in Theorem 2.1 then we get the Hadamard Integral and the above result cover Theorem 3.1 of [12].

Theorem 2.2. Let $f$ and $g$ be two synchronous functions on $[0, \infty)$. Then for all $\chi>a_{1} \geq 0, \lambda \in(0,1)$ and $\gamma, \delta \in \mathbb{C}$ with $\operatorname{Re}(\gamma)>0, \operatorname{Re}(\delta)>0$ it holds that

$$
C_{\lambda}^{\frac{\gamma}{k}, \frac{\delta}{k}}(f, g) \geq 0
$$

where

$$
C_{\lambda}^{\frac{\gamma}{k}, \frac{\delta}{k}}(f, g)=\frac{J_{F, a_{1}+}^{\frac{\gamma}{k}, \lambda}(f g)(\chi)}{J_{F, a_{1}+}^{\frac{\gamma}{k}, \lambda}(1)(\chi)}+\frac{J_{F, a_{1}+}^{\frac{\delta}{k}, \lambda}(f g)(\chi)}{J_{F, a_{1}+}^{\frac{\delta}{k}, \lambda}(1)(\chi)}-\frac{J_{F, a_{1}+}^{\frac{\delta}{k}, \lambda}(g)(\chi) J_{F, a_{1}+}^{\frac{\gamma}{k}, \lambda}(f)(\chi)+J_{F, a_{1}+}^{\frac{\delta}{k}, \lambda}(f)(\chi) J_{F, a_{1}+}^{\frac{\gamma}{k}, \lambda}(g)(\chi)}{J_{F, a_{1}+}^{\frac{\delta}{k}, \lambda}(1)(\chi) J_{F, a_{1}+}^{\frac{\gamma}{k}, \lambda}(1)(\chi)}
$$

is the generalization of the Functional $T(f, g)$ for $\gamma$ and $\delta$.

Proof. Multiplying both sides of (6) by

$$
\frac{1}{\lambda^{\delta} k \Gamma_{k}(\delta)} \frac{G\left(\mathbb{F}_{+}(\chi, v), \lambda\right) F(v)}{\left(\mathbb{F}_{+}(\chi, v)\right)^{1-\frac{\delta}{k}}},
$$

then integrating the resulting inequality with respect to $v$ over $\left(a_{1}, \chi\right)$, we obtain

$$
\begin{aligned}
& J_{F, a_{1}+}^{\frac{\delta}{k}, \lambda}(1)(\chi) J_{F, a_{1}+}^{\frac{\gamma}{k}, \lambda}(f g)(\chi)+J_{F, a_{1}+}^{\frac{\delta}{k}, \lambda}(f g)(\chi) J_{F, a_{1}+}^{\frac{\gamma}{k}, \lambda}(1)(\chi) \\
& \geq J_{F, a_{1}+}^{\frac{\delta}{k}, \lambda}(g)(\chi) J_{F, a_{1}+}^{\frac{\gamma}{k}, \lambda}(f)(\chi)+J_{F, a_{1}+}^{\frac{\delta}{k}, \lambda}(f)(\chi) J_{F, a_{1}+}^{\frac{\gamma}{k}, \lambda}(g)(\chi) .
\end{aligned}
$$


Dividing both sides of (8) by

$$
J_{F, a_{1}+}^{\frac{\delta}{k}, \lambda}(1)(\chi) J_{F, a_{1}+}^{\frac{\gamma}{k}, \lambda}(1)(\chi)
$$

we get

$$
\frac{J_{F, a_{1}+}^{\frac{\gamma}{k}, \lambda}(f g)(\chi)}{J_{F, a_{1}+}^{\frac{\gamma}{k}, \lambda}(1)(\chi)}+\frac{J_{F, a_{1}+}^{\frac{\delta}{k}, \lambda}(f g)(\chi)}{J_{F, a_{1}+}^{\frac{\delta}{k}, \lambda}(1)(\chi)} \geq \frac{J_{F, a_{1}+}^{\frac{\delta}{k}, \lambda}(g)(\chi) J_{F, a_{1}+}^{\frac{\gamma}{k}, \lambda}(f)(\chi)+J_{F, a_{1}+}^{\frac{\delta}{k}, \lambda}(f)(\chi) J_{F, a_{1}+}^{\frac{\gamma}{k}, \lambda}(g)(\chi)}{J_{F, a_{1}+}^{\frac{\delta}{k}, \lambda}(1)(\chi) J_{F, a_{1}+}^{\frac{\gamma}{k}, \lambda}(1)(\chi)}
$$

Reordering it and taking into account the definition of $C_{\lambda}^{\frac{\gamma}{k}, \frac{\delta}{k}}(f, g)$, the desired inequality is obtained. This completes the proof.

\section{Remark 2.2. Note that}

1. The setting $\gamma=\beta$ in Theorem 2.2 yields Theorem 2.1.

2. If we take $k=1$ and $\gamma=\delta=1$ in Theorem 2.2, then

$$
C_{\lambda}^{\frac{\gamma}{k}, \frac{\delta}{k}}(f, g)=T(f, g) .
$$

Theorem 2.3. Let $\left(f_{i}\right)_{i=1,2, ., n}$ be positive increasing function on $[0, \infty)$. Then for all $\chi>a_{1} \geq 0, \lambda \in(0,1)$ and $\gamma \in \mathbb{C}$ with $\operatorname{Re}(\gamma)>0$, the following inequality holds

$$
J_{F, a_{1}+}^{\frac{\gamma}{k}, \lambda}\left(\prod_{i=1}^{n} f_{i}\right)(\chi) \geq\left[J_{F, a_{1}+}^{\frac{\gamma}{k}, \lambda}(1)(\chi)\right]^{1-n} \prod_{i=1}^{n} J_{F, a_{1}+}^{\frac{\gamma}{k}, \lambda}\left(f_{i}\right)(\chi) .
$$

Proof. We prove the result by induction on $n$. Clearly, for $n=1$, it holds that

$$
J_{F, a_{1}+}^{\frac{\gamma}{k}, \lambda}\left(f_{1}\right)(\chi) \geq J_{F, a_{1}+}^{\frac{\gamma}{k}, \lambda}\left(f_{1}\right)(\chi)
$$

for all $\chi>a_{1} \geq 0, \alpha>0, \lambda \in(0,1)$ and $\gamma \in \mathbb{C}$ with $\operatorname{Re}(\gamma)>0$.

For $n=2$, applying Equation (7), we obtain

$$
J_{F, a_{1}+}^{\frac{\gamma}{k}, \lambda}\left(f_{1} f_{2}\right)(\chi) \geq\left[J_{F, a_{1}+}^{\frac{\gamma}{k}, \lambda}(1)(\chi)\right]^{-1} J_{F, a_{1}+}^{\frac{\gamma}{k}, \lambda}\left(f_{1}\right)(\chi) J_{F, a_{1}+}^{\frac{\gamma}{k}, \lambda}\left(f_{2}\right)(\chi) .
$$

By induction hypothesis, we have

$$
J_{F, a_{1}+}^{\frac{\gamma}{k}, \lambda}\left(\prod_{i=1}^{n-1} f_{i}\right)(\chi) \geq\left[J_{F, a_{1}+}^{\frac{\gamma}{k}, \lambda}(1)(\chi)\right]^{2-n} \prod_{i=1}^{n-1} J_{F, a_{1}+}^{\frac{\gamma}{k}, \lambda}\left(f_{i}\right)(\chi)
$$

for all $\chi>a_{1} \geq 0, \alpha>0, \lambda \in(0,1)$ and $\gamma \in \mathbb{C}$ with $\operatorname{Re}(\gamma)>0$.

Now, since $\left(f_{i}\right)_{i=1,2, \ldots, n}$ are positive increasing functions on $[0, \infty),\left(\prod_{i=1}^{n-1} f_{i}\right)(\chi)$ is also a positive increasing function on $[0, \infty)$. Therefore, by applying Theorem 2.1 to the functions $g=\prod_{i=1}^{n-1} f_{i}$ and $f=f_{n}$, we obtain

$$
\begin{aligned}
J_{F, a_{1}+}^{\frac{\gamma}{k}, \lambda}\left(\prod_{i=1}^{n} f_{i}\right)(\chi) & \geq J_{F, a_{1}+}^{\frac{\gamma}{k}, \lambda}\left(\prod_{i=1}^{n-1} f_{i} f_{n}\right)(\chi) J_{F, a_{1}+}^{\frac{\gamma}{k}, \lambda}(g f)(\chi) \\
& \geq\left[J_{F, a_{1}+}^{\frac{\gamma}{k}, \lambda}(1)(\chi)\right]^{-1} J_{F, a_{1}+}^{\frac{\gamma}{k}, \lambda}(g)(\chi) J_{F, a_{1}+}^{\frac{\gamma}{k}, \lambda}(f)(\chi) \\
& \geq\left[J_{F, a_{1}+}^{\frac{\gamma}{k}, \lambda}(1)(\chi)\right]^{-1} J_{F, a_{1}+}^{\frac{\gamma}{k}, \lambda}\left(\prod_{i=1}^{n-a}\right)(\chi) J_{F, a_{1}+}^{\frac{\gamma}{k}, \lambda}\left(f_{n}\right)(\chi) \\
& \geq\left[J_{F, a_{1}+}^{\frac{\gamma}{k}, \lambda}(1)(\chi)\right]^{-1}\left[J_{F, a_{1}+}^{\frac{\gamma}{k}, \lambda}(1)(\chi)\right]^{2-n} \prod_{i=1}^{n-1} J_{F, a_{1}+}^{\frac{\gamma}{k}, \lambda}\left(f_{i}\right)(\chi) J_{F, a_{1}+}^{\frac{\gamma}{k}, \lambda}\left(f_{n}\right)(\chi) \\
& \geq\left[J_{F, a_{1}+}^{\frac{\gamma}{k}, \lambda}(1)(\chi)\right]^{1-n} \prod_{i=1}^{n} J_{F, a_{1}+}^{\frac{\gamma}{k}, \lambda}\left(f_{i}\right)(\chi) .
\end{aligned}
$$

This completes the proof. 
The previous results can be extended if we consider a certain positive "weight" function $h$.

Theorem 2.4. Let $f$ and $g$ be two synchronous functions on $[0, \infty)$ and $h \geq 0$. Then for all $\chi>a_{1} \geq 0, \lambda>0$ and $\gamma \in \mathbb{C}$ with $\operatorname{Re}(\gamma)>0$, the following inequality holds:

$$
\begin{aligned}
J_{F, a_{1}+}^{\frac{\gamma}{k}, \lambda}(1)(\chi) J_{F, a_{1}+}^{\frac{\gamma}{k}, \lambda}(f g h)(\chi) \geq & J_{F, a_{1}+}^{\frac{\gamma}{k}, \lambda}(g)(\chi) J_{F, a_{1}+}^{\frac{\gamma}{k}, \lambda}(f h)(\chi)+J_{F, a_{1}+}^{\frac{\gamma}{k}, \lambda}(f)(\chi) J_{F, a_{1}+}^{\frac{\gamma}{k}, \lambda}(g h)(\chi) \\
& -J_{F, a_{1}+}^{\frac{\gamma}{k}, \lambda}(f g)(\chi) J_{F, a_{1}+}^{\frac{\gamma}{k}, \lambda}(h)(\chi) .
\end{aligned}
$$

Proof. Since $h \geq 0$ and the functions $f$ and $g$ are synchronous on $[0, \infty)$, we obtain

$$
(f(u)-f(v))(g(u)-g(v))(h(u)+h(v)) \geq 0,
$$

for all $u, v \geq 0$. Then,

$$
\begin{aligned}
f(u) g(u) h(u)+f(v) g(v) h(v) \geq & f(u) g(v) h(u)+f(v) g(u) h(u)-f(v) g(v) h(u)-f(u) g(u) h(v) \\
& +f(u) g(v) h(v)+f(v) g(u) h(v) .
\end{aligned}
$$

Multiplying both sides of (9) by

$$
\frac{1}{\lambda^{\gamma} k \Gamma_{k}(\gamma)} \frac{G\left(\mathbb{F}_{+}(\chi, u), \lambda\right) F(u)}{\left(\mathbb{F}_{+}(\chi, u)\right)^{1-\frac{\gamma}{k}}},
$$

then integrating the resulting inequality with respect to $u$ over $\left(a_{1}, \chi\right)$, we obtain

$$
\begin{aligned}
& \frac{1}{\lambda^{\gamma} k \Gamma_{k}(\gamma)} \int_{a_{1}}^{\chi} \frac{G\left(\mathbb{F}_{+}(\chi, u), \lambda\right) F(u) f(u) g(u) h(u) d u}{\left(\mathbb{F}_{+}(\chi, u)\right)^{1-\frac{\gamma}{k}}}+\frac{f(v) g(v) h(v)}{\lambda^{\gamma} k \Gamma_{k}(\gamma)} \int_{a_{1}}^{\chi} \frac{G\left(\mathbb{F}_{+}(\chi, u), \lambda\right) F(u) d u}{\left(\mathbb{F}_{+}(\chi, u)\right)^{1-\frac{\gamma}{k}}} \\
\geq & \frac{g(v)}{\lambda^{\gamma} k \Gamma_{k}(\gamma)} \int_{a_{1}}^{\chi} \frac{G\left(\mathbb{F}_{+}(\chi, u), \lambda\right) F(u) f(u) h(u) d u}{\left(\mathbb{F}_{+}(\chi, u)\right)^{1-\frac{\gamma}{k}}}+\frac{f(v)}{\lambda^{\gamma} k \Gamma_{k}(\gamma)} \int_{a_{1}}^{\chi} \frac{G\left(\mathbb{F}_{+}(\chi, u), \lambda\right) F(u) g(u) h(u) d u}{\left(\mathbb{F}_{+}(\chi, u)\right)^{1-\frac{\gamma}{k}}} \\
& -\frac{f(v) g(v)}{\lambda^{\gamma} k \Gamma_{k}(\gamma)} \int_{a_{1}}^{\chi} \frac{G\left(\mathbb{F}_{+}(\chi, u), \lambda\right) F(u) h(u) d u}{\left(\mathbb{F}_{+}(\chi, u)\right)^{1-\frac{\gamma}{k}}}-\frac{h(v)}{\lambda^{\gamma} k \Gamma_{k}(\gamma)} \int_{a_{1}}^{\chi} \frac{G\left(\mathbb{F}_{+}(\chi, u), \lambda\right) F(u) f(u) g(u) d u}{\left(\mathbb{F}_{+}(\chi, u)\right)^{1-\frac{\gamma}{k}}} \\
& +\frac{g(v) h(v)}{\lambda^{\gamma} k \Gamma_{k}(\gamma)} \int_{a_{1}}^{\chi} \frac{G\left(\mathbb{F}_{+}(\chi, u), \lambda\right) F(u) f(u) d u}{\left(\mathbb{F}_{+}(\chi, u)\right)^{1-\frac{\gamma}{k}}}+\frac{f(v) h(v)}{\lambda^{\gamma} k \Gamma_{k}(\gamma)} \int_{a_{1}}^{\chi} \frac{G\left(\mathbb{F}_{+}(\chi, u), \lambda\right) F(u) g(u) d u}{\left(\mathbb{F}_{+}(\chi, u)\right)^{1-\frac{\gamma}{k}}}
\end{aligned}
$$

We can write as

$$
\begin{aligned}
J_{F, a_{1}+}^{\frac{\gamma}{k}, \lambda}(f g h)(\chi)+f(v) g(v) h(v) J_{F, a_{1}+}^{\frac{\gamma}{k}, \lambda}(1)(\chi) \geq & g(v) J_{F, a_{1}+}^{\frac{\gamma}{k}, \lambda}(f h)(\chi)+f(v) J_{F, a_{1}+}^{\frac{\gamma}{k}, \lambda}(g h)(\chi) \\
& -f(v) g(v) J_{F, a_{1}+}^{\frac{\gamma}{k}, \lambda}(h)(\chi)-h(v) J_{F, a_{1}+}^{\frac{\gamma}{k}, \lambda}(f g)(\chi) \\
& +g(v) h(v) J_{F, a_{1}+}^{\frac{\gamma}{k}, \lambda}(f)(\chi) \\
& +f(v) h(v) J_{F, a_{1}+}^{\frac{\gamma}{k}, \lambda}(g)(\chi) .
\end{aligned}
$$

Multiplying both sides of (10) by

$$
\frac{1}{\lambda^{\gamma} k \Gamma_{k}(\gamma)} \frac{G\left(\mathbb{F}_{+}(\chi, v), \lambda\right) F(v)}{\left(\mathbb{F}_{+}(\chi, v)\right)^{1-\frac{\gamma}{k}}},
$$

then integrating the resulting inequality with respect to $v$ over $\left(a_{1}, \chi\right)$, we obtain

$$
\begin{aligned}
J_{F, a_{1}+}^{\frac{\gamma}{k}, \lambda}(1)(\chi) J_{F, a_{1}+}^{\frac{\gamma}{k}, \lambda}(f g h)(\chi)+ & J_{F, a_{1}+}^{\frac{\gamma}{k}, \lambda}(f g h)(\chi) J_{F, a_{1}+}^{\frac{\gamma}{k}, \lambda}(1)(\chi) \geq J_{F, a_{1}+}^{\frac{\gamma}{k}, \lambda}(g)(\chi) J_{F, a_{1}+}^{\frac{\gamma}{k}, \lambda}(f h)(\chi) \\
& +J_{F, a_{1}+}^{\frac{\gamma}{k}, \lambda}(f)(\chi) J_{F, a_{1}+}^{\frac{\gamma}{k}, \lambda}(g h)(\chi)-J_{F, a_{1}+}^{\frac{\gamma}{k}, \lambda}(f g)(\chi) J_{F, a_{1}+}^{\frac{\gamma}{k}, \lambda}(h)(\chi) \\
& -J_{F, a_{1}+}^{\frac{\gamma}{k}, \lambda}(h)(\chi) J_{F, a_{1}+}^{\frac{\gamma}{k}, \lambda}(f g)(\chi)+J_{F, a_{1}+}^{\frac{\gamma}{k}, \lambda}(g h)(\chi) J_{F, a_{1}+}^{\frac{\gamma}{k}, \lambda}(f)(\chi) \\
& +J_{F, a_{1}+}^{\frac{\gamma}{k}, \lambda}(f h)(\chi) J_{F, a_{1}+}^{\frac{\gamma}{k}, \lambda}(g)(\chi) .
\end{aligned}
$$

That is,

$$
J_{F, a_{1}+}^{\frac{\gamma}{k}, \lambda}(1)(\chi) J_{F, a_{1}+}^{\frac{\gamma}{k}, \lambda}(f g h)(\chi) \geq J_{F, a_{1}+}^{\frac{\gamma}{k}, \lambda}(g)(\chi) J_{F, a_{1}+}^{\frac{\gamma}{k}, \lambda}(f h)(\chi)+J_{F, a_{1}+}^{\frac{\gamma}{k}, \lambda}(f)(\chi) J_{F, a_{1}+}^{\frac{\gamma}{k}, \lambda}(g h)(\chi)-J_{F, a_{1}+}^{\frac{\gamma}{k}, \lambda}(f g)(\chi) J_{F, a_{1}+}^{\frac{\gamma}{k}, \lambda}(h)(\chi) .
$$


Remark 2.3. If we take $h=1$ in Theorem 2.4, we obtain Theorem 2.1.

Theorem 2.5. Let $f$ and $g$ be two synchronous functions on $[0, \infty), h \geq 0$. Then for all $\chi>a_{1} \geq 0, \lambda \in(0,1), \gamma \in \mathbb{C}$ with $\operatorname{Re}(\gamma)>0$ and $\delta \in \mathbb{C}$ with $\operatorname{Re}(\delta)>0$, the following inequality holds:

$$
\begin{aligned}
& J_{F, a_{1}+}^{\frac{\delta}{k}, \lambda}(h)(\chi) J_{F, a_{1}+}^{\frac{\gamma}{k}, \lambda}(f g h)(\chi)+J_{F, a_{1}+}^{\frac{\delta}{k}, \lambda}(f g h)(\chi) J_{F, a_{1}+}^{\frac{\gamma}{k}, \lambda}(h)(\chi) \\
\geq & J_{F, a_{1}+}^{\frac{\delta}{k}, \lambda}(g h)(\chi) J_{F, a_{1}+}^{\frac{\gamma}{k}, \lambda}(f h)(\chi)+J_{F, a_{1}+}^{\frac{\delta}{k}, \lambda}(f h)(\chi) J_{F, a_{1}+}^{\frac{\gamma}{k}, \lambda}(g h)(\chi) .
\end{aligned}
$$

Proof. Multiplying both sides of (10) by

$$
\frac{1}{\lambda^{\delta} k \Gamma_{k}(\delta)} \frac{G\left(\mathbb{F}_{+}(\chi, v), \lambda\right) F(v)}{\left(\mathbb{F}_{+}(\chi, v)\right)^{1-\frac{\delta}{k}}},
$$

then integrating the resulting inequality with respect to $v$ over $\left(a_{1}, \chi\right)$, we obtain

$$
\begin{aligned}
J_{F, a_{1}+}^{\frac{\delta}{k}, \lambda}(1)(\chi) J_{F, a_{1}+}^{\frac{\gamma}{k}, \lambda}(f g h)(\chi)+ & J_{F, a_{1}+}^{\frac{\delta}{k}, \lambda}(f g h)(\chi) J_{F, a_{1}+}^{\frac{\gamma}{k}, \lambda}(1)(\chi) \geq J_{F, a_{1}+}^{\frac{\delta}{k}, \lambda}(g)(\chi) J_{F, a_{1}+}^{\frac{\gamma}{k}, \lambda}(f h)(\chi) \\
& +J_{F, a_{1}+}^{\frac{\delta}{k}, \lambda}(f)(\chi) J_{F, a_{1}+}^{\frac{\gamma}{k}, \lambda}(g h)(\chi)-J_{F, a_{1}+}^{\frac{\delta}{k}, \lambda}(f g)(\chi) J_{F, a_{1}+}^{\frac{\gamma}{k}, \lambda}(h)(\chi) \\
& -J_{F, a_{1}+}^{\frac{\delta}{k}, \lambda}(h)(\chi) J_{F, a_{1}+}^{\frac{\gamma}{k}, \lambda}(f g)(\chi)+J_{F, a_{1}+}^{\frac{\delta}{k}, \lambda}(g h)(\chi) J_{F, a_{1}+}^{\frac{\gamma}{k}, \lambda}(f)(\chi) \\
& +J_{F, a_{1}+}^{\frac{\delta}{k}, \lambda}(f h)(\chi) J_{F, a_{1}+}^{\frac{\gamma}{k}, \lambda}(g)(\chi) .
\end{aligned}
$$

Remark 2.4. For $h=1$, Theorem 2.5 gives Theorem 2.2.

More precise results can be obtained, if in the previous Theorem we impose additional conditions on the function $h$.

Theorem 2.6. Let $f, g$ and $h$ be three monotonic functions defined on $[0, \infty)$ satisfying the following inequality

$$
(f(u)-f(v))(g(u)-g(v))(h(u)-h(v)) \geq 0
$$

for all $u, v \in\left[a_{1}, \chi\right]$. Then, for all $\chi>a_{1} \geq 0, \lambda \in(0,1), \gamma \in \mathbb{C}$ with $\operatorname{Re}(\gamma)>0$ and $\delta \in \mathbb{C}$ with $\operatorname{Re}(\delta)>0$, it holds that

$$
\begin{aligned}
J_{F, a_{1}+}^{\frac{\delta}{k}, \lambda}(1)(\chi) J_{F, a_{1}+}^{\frac{\gamma}{k}, \lambda}(f g h)(\chi)+ & J_{F, a_{1}+}^{\frac{\delta}{k}, \lambda}(f g h)(\chi) J_{F, a_{1}+}^{\frac{\gamma}{k}, \lambda}(1)(\chi) \geq J_{F, a_{1}+}^{\frac{\delta}{k}, \lambda}(g)(\chi) J_{F, a_{1}+}^{\frac{\gamma}{k}, \lambda}(f h)(\chi) \\
& +J_{F, a_{1}+}^{\frac{\delta}{k}, \lambda}(f)(\chi) J_{F, a_{1}+}^{\frac{\gamma}{k}, \lambda}(g h)(\chi)-J_{F, a_{1}+}^{\frac{\delta}{k}, \lambda}(f g)(\chi) J_{F, a_{1}+}^{\frac{\gamma}{k}, \lambda}(h)(\chi) \\
& -J_{F, a_{1}+}^{\frac{\delta}{k}, \lambda}(h)(\chi) J_{F, a_{1}+}^{\frac{\gamma}{k}, \lambda}(f g)(\chi)+J_{F, a_{1}+}^{\frac{\delta}{k}, \lambda}(g h)(\chi) J_{F, a_{1}+}^{\frac{\gamma}{k}, \lambda}(f)(\chi) \\
& +J_{F, a_{1}+}^{\frac{\delta}{k}, \lambda}(f h)(\chi) J_{F, a_{1}+}^{\frac{\gamma}{k}, \lambda}(g)(\chi) .
\end{aligned}
$$

Proof. As in the proof of Theorem 2.5, if we multiply both sides of (10) by

$$
\frac{1}{\lambda^{\delta} k \Gamma_{k}(\delta)} \frac{G\left(\mathbb{F}_{+}(\chi, v), \lambda\right) F(v)}{\left(\mathbb{F}_{+}(\chi, v)\right)^{1-\frac{\delta}{k}}},
$$

then integrate the resulting inequality with respect to $v$ over $\left(a_{1}, \chi\right)$, we obtain the desired inequality.

An inequality involving the square of the functions $f$ and $g$ can be stated as follows.

Theorem 2.7. Let $f$ and $g$ be defined on $[0, \infty)$, then for all $\chi>a_{1} \geq 0, \lambda \in(0,1)$ and $\gamma \in \mathbb{C}$ with Re $(\gamma)>0$, the following inequalities hold

$$
J_{F, a_{1}^{+}}^{\frac{\gamma}{k}, \lambda}(1)\left[J_{F, a_{1}^{+}}^{\frac{\gamma}{k}, \lambda} f^{2}(\chi)+J_{F, a_{1}^{+}}^{\frac{\gamma}{k}, \lambda} g^{2}(\chi)\right] \geq 2 J_{F, a_{1}^{+}}^{\frac{\gamma}{k}, \lambda} f(\chi) J_{F, a_{1}^{+}}^{\frac{\gamma}{k}, \lambda} g(\chi)
$$

and

Proof. Since

$$
J_{F, a_{1}^{+}}^{\frac{\gamma}{k}, \lambda} f^{2}(\chi) J_{F, a_{1}^{+}}^{\frac{\gamma}{k}, \lambda} g^{2}(\chi) \geq\left[J_{F, a_{1}^{+}}^{\frac{\gamma}{k}, \lambda}(f g)(\chi)\right]^{2} .
$$

$$
(f(u)-g(v))^{2} \geq 0,
$$

we have

$$
f^{2}(u)+g^{2}(v) \geq 2 f(u) g(v) .
$$


Multiplying both sides of (13) by

$$
\frac{1}{\lambda^{\gamma} k \Gamma_{k}(\gamma)} \frac{G\left(\mathbb{F}_{+}(\chi, u), \lambda\right) F(u)}{\left(\mathbb{F}_{+}(\chi, u)\right)^{1-\frac{\gamma}{k}}},
$$

then integrating the resulting inequality with respect to $u$ and $v$ over $\left(a_{1}, \chi\right)$, we obtain (11).

On the other hand, since

$$
(f(u) g(v)-f(v) g(u))^{2} \geq 0
$$

then by using the same arguments as before, we have (12).

Theorem 2.8. Let $f$ and $g$ be defined on $[0, \infty)$. Then for all $\chi>a_{1} \geq 0, \lambda \in(0,1), \gamma \in \mathbb{C}$ with Re( $\left.\gamma\right)>0$ and $\delta \in \mathbb{C}$ with $\operatorname{Re}(\delta)>0$, the following inequalities hold

$$
J_{F, a_{1}^{+}}^{\frac{\gamma}{k}, \lambda} f^{2}(\chi) J_{F, a_{1}^{+}}^{\frac{\delta}{k}, \lambda}(1)+J_{F, a_{1}^{+}}^{\frac{\gamma}{k}, \lambda}(1) J_{F, a_{1}^{+}}^{\frac{\delta}{k}, \lambda} g^{2}(\chi) \geq 2 J_{F, a_{1}^{+}}^{\frac{\gamma}{k}, \lambda} f(\chi) J_{F, a_{1}^{+}}^{\frac{\delta}{k}, \lambda} g(\chi)
$$

and

$$
J_{F, a_{1}^{+}}^{\frac{\gamma}{k}, \lambda} f^{2}(\chi) J_{F, a_{1}^{+}}^{\frac{\delta}{k}, \lambda} g^{2}(\chi)+J_{F, a_{1}^{+}}^{\frac{\delta}{k}} f^{2}(\chi) J_{F, a_{1}^{+}}^{\frac{\gamma}{k}, \lambda} g^{2}(\chi) \geq 2 J_{F, a_{1}^{+}}^{\frac{\gamma}{k}, \lambda}(f g)(\chi) J_{F, a_{1}^{+}}^{\frac{\delta}{k}, \lambda}(f g)(\chi) .
$$

Proof. Like before, since $(f(u)-g(v))^{2} \geq 0$, we have

$$
f^{2}(u)+g^{2}(v) \geq 2 f(u) g(v)
$$

Multiplying both sides of (16) by

and

$$
\frac{1}{\lambda^{\gamma} k \Gamma_{k}(\gamma)} \frac{G\left(\mathbb{F}_{+}(\chi, u), \lambda\right) F(u)}{\left(\mathbb{F}_{+}(\chi, u)\right)^{1-\frac{\gamma}{k}}}
$$

$$
\frac{1}{\lambda^{\delta} k \Gamma_{k}(\delta)} \frac{G\left(\mathbb{F}_{+}(\chi, v), \lambda\right) F(v)}{\left(\mathbb{F}_{+}(\chi, v)\right)^{1-\frac{\delta}{k}}},
$$

then integrating the resulting inequality with respect to $u$ and $v$ over $\left(a_{1}, \chi\right)$ respectively, we obtain (14).

On the other hand, since

$$
(f(u) g(v)-f(v) g(u))^{2} \geq 0
$$

then by using the same arguments as before, we have (15).

Remark 2.5. If we take $\gamma=\delta$ we obtain Theorem 2.7.

Theorem 2.9. Let $f: \mathbb{R} \rightarrow \mathbb{R}$ with

$$
\bar{f}(u)=\int_{a_{1}}^{u} F(z, s) f(z) d z
$$

$u>a_{1} \geq 0, \lambda \in(0,1)$ and $\gamma \in \mathbb{C}$ with $\operatorname{Re}(\gamma)>0$. Then, for $\gamma \geq k>0$ it holds that

$$
J_{F, a_{1}^{+}}^{\frac{\gamma+k}{k}, \lambda} f(\chi)=\frac{1}{k} J_{F, a_{1}}^{\frac{\gamma}{k}, \lambda} \bar{f}(\chi) .
$$

Proof. Here

$$
\begin{aligned}
J_{F, a_{1}^{+}}^{\frac{\gamma}{k}, \lambda} \bar{f}(\chi) & =\frac{1}{\lambda^{\gamma} k \Gamma_{k}(\gamma)} \int_{a_{1}}^{\chi} \frac{G\left(\mathbb{F}_{+}(\chi, u), \lambda\right) F(u) \bar{f}(u)}{\left(\mathbb{F}_{+}(\chi, u)\right)^{1-\frac{\gamma}{k}}} d u \\
& =\frac{1}{\lambda^{\gamma} k \Gamma_{k}(\gamma)} \int_{a_{1}}^{\chi} \frac{G\left(\mathbb{F}_{+}(\chi, u), \lambda\right) F(u)}{\left(\mathbb{F}_{+}(\chi, u)\right)^{1-\frac{\gamma}{k}}} \int_{a_{1}}^{u} F(z, s) f(z) d z d u .
\end{aligned}
$$

Then, by Dirichlet's formula, the last expression becomes

$$
\begin{aligned}
& \frac{1}{\lambda^{\gamma} k \Gamma_{k}(\gamma)} \int_{a_{1}}^{\chi} F(z, s) f(z) \int_{z}^{\chi} G\left(\mathbb{F}_{+}(\chi, u), \lambda\right) F(u)\left(\mathbb{F}_{+}(\chi, u)\right)^{\frac{\gamma}{k}-1} d u d z \\
& =\frac{1}{\lambda^{\gamma} \Gamma_{k}(\gamma+k)} \int_{a_{1}}^{\chi} \frac{G\left(\mathbb{F}_{+}(\chi, u), \lambda\right) F(u) f(u)}{\left(\mathbb{F}_{+}(\chi, u)\right)^{1-\frac{\gamma+k}{k}}} \\
& =k J_{F, a_{1}^{+}}^{\frac{\gamma+k}{k}} f(u) .
\end{aligned}
$$

This complete the proof. 


\section{Conclusion}

In this paper, we present a generalized formulation of the Riemann-Liouville fractional integral, which contains as particular cases many of the integral operators reported in the literature. We present several integral inequalities that generalize several known inequalities.

We highlight the strength of Definition 1.9 by pointing out the following fact. If we consider the kernel

$$
F(\chi, s)=\chi^{1-s}
$$

and $G \equiv 1$, we obtain a variant of the $(k, s)$-Riemann-Liouville fractional integral defined in [40]:

$$
{ }_{a_{1}}^{s} I_{u}^{\alpha} f(u)=\frac{(2-s)^{1-\frac{\alpha}{k}}}{k \Gamma_{k}(\alpha)} \int_{a_{1}}^{u}\left(u^{2-s}-\chi^{2-s}\right)^{\frac{\alpha}{k}-1} \chi^{1-s} f(\chi) d \chi .
$$

This opens up wide possibilities of obtaining new integral inequalities.

\section{Acknowledgment}

The authors express their gratitude to the anonymous referees and editor for their comments that helped to improve the earlier version of this paper.

\section{References}

[1] T. Abdeljawad, On conformable fractional calculus, J. Comput. Appl. Math. 279 (2015) 57-66.

[2] P. Agarwal, J. Tariboon, S. K. Ntouyas, Some generalized Riemann-Liouville $k$-fractional integral inequalities, J. Inequal. Appl. 2016 (2016) \#122.

[3] B. Ahmad, A. Alsaedi, M. Kirane, B. T. Toberek, Hermite-Hadamard, Hermite-Hadamard-Fejer, Dragomir-Agarwal and Pachpatte type inequalities for convex functions via new fractional integrals, J. Comput. Appl. Math. 353 (2019) 120-129.

[4] A. Akkurt, M. E. Yildirim, H. Yildirim, On some integral inequalities for ( $k, h)$-Riemann-Liouville fractional integral, New Trends Math. Sci. 4 (2016) 138-146.

[5] M. A. Ali, J. E. Nápoles Valdés, A. Kashuri, Z. Zhang, Fractional non conformable Hermite-Hadamard inequalities for generalized $\phi$-convex functions, Fasc. Math. 64 (2020) 5-16.

[6] A. Atangana, Derivative with a New Parameter Theory, Methods and Applications, Academic Press, London, 2016.

[7] D. Baleanu, Comments on: "The failure of certain fractional calculus operators in two physical models" by M. Ortigueira, V. Martynyuk, M. Fedula and J.A.T. Machado, Fract. Calc. Appl. Anal. 23 (2020) 292-297.

[8] D. Baleanu, A. Fernandez, On fractional operators and their classifications, Mathematics 7 (2019) \#830.

[9] D. Baleanu, S. D. Purohit, Chebyshev type integral inequalities involving the fractional hypergeometric operators, Abstr. Appl. Anal. 2014 (2014) \#609160.

[10] S. Bermudo, P. Kórus, J. E. Nápoles Valdés, On $q$-Hermite-Hadamard inequalities for general convex functions, Acta Math. Hungar. 162 (2020) 364-374.

[11] P. L. Chebyshev, Sur les expressions approximatives des integrales definies par les autres prise entre les memes limites, Proc. Sco. Charkov 2 (1882) 93-98.

[12] V. L. Chinchane, D. B. Pachpatte, A note on some fractional integral inequalities via Hadamard integral, J. Fract. Calc. Appl. 4 (2013) 125-129.

[13] R. Díaz, E. Pariguan, On hypergeometric functions and Pochhammer k-symbol, Divulg. Mat. 15 (2007) 179-192.

[14] R. S. Dubey, P. Goswami, Some fractional integral inequalities for the Katugampola integral operator, AIMS Math. $4193-198$.

[15] J. Galeano, J. E. Nápoles Valdés, E. Pérez, A note on some integral inequalities in a generalized framework, Int. J. Appl. Math. Stat. 60 (2021) $45-52$.

[16] R. Gorenflo, F. Mainardi, Fractals and Fractional Calculus in Continuum Mechanics, Springer, Vienna, 1997, pp. 223-276.

[17] P. M. Guzmán, P. Kórus, J. E. Nápoles Valdés, Generalized integral inequalities of Chebyshev type, Fractal Fract. 4 (2020) \#10.

[18] P. M. Guzmán, J. E. Nápoles Valdés, Generalized fractional Grüss-type inequalities, Contrib. Math. 2 (2020) $16-21$.

[19] P. R. Halmos, Measure Theory, D. Van Nostrand Company, New York, 1950.

[20] S. Jain, P. Agarwal, B. Ahmad, S. K. Q. Al-Omari, Certain recent fractional integral inequalities associated with the hypergeometric operators, J. King Saud Univ. Sci. 28 (2016) 82-86.

[21] F. Jarad, E. Uğurlu, T. Abdeljawad, D. Baleanu, On a new class of fractional operators, Adv. Difference Equ. 2017 (2017) \#247.

[22] U. N. Katugampola, New approach generalized fractional integral, Appl. Math. Comput. 218 (2011) 860-865.

[23] A. A. Kilbas, O. I. Marichev, S. G. Samko, Fractional Integrals and Derivatives: Theory and Applications, Gordon \& Breach, Yverdon, 1993.

[24] A. A. Kilbas, H. M. Srivastava, J. J. Trujillo, Theory and Applications of Fractional Differential Equations, Elsevier, New York, 2006.

[25] P. Kórus, L. M. Lugo Motta, J. E. Nápoles Valdés, Integral inequalities in a generalized context, Studia Sci. Math. Hungar. 57 (2020) 312-320.

[26] Y. C. Kwun, G. Farid, W. Nazeer, S. Ullah, S. M. Kang, Generalized Riemann-Liouville $k$-fractional integrals associated with Ostrowski type inequalities and error bounds of Hadamard inequalities, IEEE Access 6 (2018) 64946-64953.

[27] Z. Liu, W. Yang, P. Agarwal, Certain Chebyshev type inequalities involving the generalized fractional integral operator, J. comput. Anal. Appl. 22 (2017) 999-1014.

[28] S. Mubeen, G. M. Habibullah, $k$-Fractional integrals and applications, Int. J. Contemp. Math. Sci. 7 (2012) 89-94.

[29] S. Mubeen, G. M. Habibullah, M. N. Naeem, The Minkowski inequality involving generalized $k$-fractional conformable integral, J. Inequal. Appl. 2019 (2019) \#81.

[30] J. E. Nápoles Valdés, A generalized $k$-proportional fractional integral operators with general kernel, Submited.

[31] J. E. Nápoles Valdés, J. M. Rodríguez, J. M. Sigarreta, New Hermite-Hadamard type inequalities involving non-conformable integral operators, Symmetry 11 (2019) \#1108.

[32] M. E. Özdemir, E. Set, A. O. Akdemir, M. Z. Sarikaya, Some new Chebyshev type inequalities for functions whose derivatives belongs to $L_{p}$ spaces, Afrika Mat. 26 (2015) 1609-1619.

[33] F. Qi, B. N. Guo, Integral representations and complete monotonicity of remainders of the Binet and Stirling formulas for the gamma function, Rev. R. Acad. Cienc. Exactas Fís. Nat. Ser. A Mat. 111 (2017) 425-434.

[34] F. Qi, S. Habib, S. Mubeen, M. N. Naeem, Generalized $k$-fractional conformable integrals and related inequalities, AIMS Math. 4 (2019) $343-358$.

[35] G. Rahman, T. Abdeljawad, F. Jarad, A. Khan, K. S. Nisar, Certain inequalities via generalized proportional Hadamard fractional integral operators, Adv. Difference Equ. 2019 (2019) \#454. 
[36] E. D. Rainville, Special Functions, Macmillan Co., New York, 1960.

[37] S. Rashid, Z. Hammouch, H. Kalsoom, R. Ashraf, Y. M. Chu, New investigation on the generalized $k$-fractional integral operators, Front. Phys. 8 (2020) \#25.

[38] S. Rashid, F. Jarad, M. A. Noor, H. Kalsoom, Y. M. Chu, Inequalities by means of generalized proportional fractional integral operators with respect to another function, Mathematics 7 (2020) \#1225.

[39] S. G. Samko, A. A. Kilbas, O. I. Marichev, Fractional Integrals and Derivatives, Gordon \& Breach Science, Yverdon, 1993.

[40] M. Z. Sarikaya, Z. Dahmani, M. E. Kiris, F. Ahmad, $(k, s)$-Riemann-Liouville fractional integral and applications, Hacet. J. Math. Stat. 45 (2016) $77-89$.

[41] E. Set, J. Choi, I. Mumcu, Chebyshev type inequalities involving generalized Katugampola fractional integral operators, Tamkang J. Math. 50 (2019) 381-390.

[42] E. Set, Z. Dahmani, I. Mumcu, New extensions of Chebyshev type inequalities using generalized Katugampola integrals via Pólya-Szegö inequality, Int. J. Optim. Control. Theor. Appl. 8 (2018) 137-144.

[43] E. Set, M. E. Özdemir, S. Demirbaş, Chebyshev type inequalities involving extended generalized fractional integral operators, AIMS Math. 5 (2020) $3573-3583$.

[44] S. Umarov, S. Steinberg, Variable order differential equations with piecewise constant order-function and diffusion with changing modes, Z. Anal. Anwend. 28 (2009) 431-450.

[45] W. Yang, Some new Chebyshev and Grüss-type integral inequalities for Saigo fractional integral operators and their $q$-analogues, Filomat 29 (2015) $1269-1289$.

[46] Z. H. Yang, J. F. Tian, Monotonicity and inequalities for the gamma function, J. Inequal. Appl. 2017 (2017) \#317.

[47] Z. H. Yang, J. F. Tian, Monotonicity and sharp inequalities related to gamma function, J. Math. Inequal. 12 (2018) 1-22. 